\title{
OBSERVATIONS SUR Saprolegnia australis ELLIOTT, AGENT PATHOGENE DE LA SAPROLEGNIOSE DES POISSONS
}

\author{
B. TH. PAPATHEODOROU \\ Direction des Services Vétérinaires du departement d'Achaïa, \\ Karolou 38. PATRAS, Grèce
}

\begin{abstract}
RESUME
Saprolegnia australis n'a jamais été rapporte comme cause primaire de la Saprolegniose chez les poissons et son pouvoir pathogène n'a jámais été vérifié par inoculation expérimentale. Nous l'avons isolé sur des gardons (Rutilus rutilus L.) atteints d'une mycose et nous l'avons inoculé avec succès à des poisson; exotiques. Nous avons ainsi vérifié le potentiel pathogène de cette espèce de champignon et pu établir avec certitude une causalité entre la seule présence de $S$. australis et la Saprolegniose observée.
\end{abstract}

\section{SUMMARY}

Saprolegnia australis has never been reported to be the primary cause of the saprolegnosis among fish and its pathogenic capacity has never been tested through experimental inoculation. We managed to isolate it on roaches affected with a mycosis and we successfully inoculated it on exotic fish. Consequently we checked the pathogenic potential of this species of fungoid growth and managed to establish undoubtedly a causality between the actual presence of Saprolegnia australis and the observed saprolegnosis.

\section{INTRODUCTION}

Dans les recherches sur la saprolegniose des poissons les premières études mycologiques apparaissent au début de notre siècle et on note, $t=n$ 1923. un travail fondamental de COKER sur l'étude et la détermination des Saprolegniaceae. Depuis, la litterature ichtyomycophathologique ne mentionne pratiquement que le genr. Saprolegnia dans son ensemble et, dans la plupart de ces rapports, manquent la description des espèces et la verification du pouvoir pathogène des isolats obtenus. Cependant d'autres genres ont été rencontrés à l'état parasite sur des animaux aquatiques et civers auteurs ont mis en évidence la relation existant entre les maladies des poissons et les espèces des divers genres de champignons Saprolegniaceae : le genre Achlya, mais également les genres Aphanomyces, Dichthyuchus, Leptomitus, Pythium et Allomyces (TIFFNEY, 1939 ; SCOTT et O'BIER, 1962 ; SRIVASTAVA et SRIVASTAVA, 1976, 1977, 1978)

En ce qui concerne le genre Saprolegnia, depuis le travail de COKER et jusqu'à il y a quelques annèes, il y avait une forte tendance à considerer que la saprolegniose doit gènéralement être attribuée à $S$. parasitica. Aujourd'hui, diverses espèces du genre Saprolegnia ont été impliquées dans la saprolegniose naturelle $\epsilon_{i}$ expérimentale des poissons (tableaux 1 et 2), mais $S$. australis n'a jamais èté signalé comme agent pathogène unique, il a toujours été trouvé en association et par conséquent impliqué dans des atteintes par un * complexe fongique.

Le but du présent travail a été d'une part de determiner précisèment l'espèce de champignon parasite, d'autre part de verifier par inoculation experiinentale son pouvoir pathogène.

\section{MATERIEL ET METHODES}

Les observations se rapportent à des gardons (Rutilus rutilus L.) appartenant à un lot en élevage dans un bassin expérimental de l'Ecole Nationale Superieure Agronomique de Toulouse (France). Les poissons étaient touchés par la saprolegniose au niveau de la zone caudale.

\section{- Mycologie}

Les fragments de champignons prélevés sur les poissons malades sont cultives dans l'eau distillée stérile en boite de Petri à la températude de $18^{\circ} \mathrm{C}$ sur graines de chanvre bouillies. Apres apparition des colonies, les cultures sont ensuite purifiées par repiquages successifs et suivant la méthode de RAPER (1937) sur Corn-Meal-Agar. Enfin, pour avoir une monoculture, nous avons realisé des cultures monosporées selon JOHNSON (1956). Pour l'identification de nos souches isolées, nous nous sommes basés sur le travail de SEYMOUR (1970) 
Tableau 1 : Espèces de Saprolegnia connues à l'état parasite sur les poissons et leurs œufs.

\begin{tabular}{|l|l|}
\hline $\begin{array}{l}\text { (S. delica) } \\
\text { S. diclina }\end{array}$ & $\begin{array}{l}\text { MCKEY (1967), NOLARD-TINTIGNER (1970). WILLOUGHBY } \\
\text { (1970), SRIVASTAVA et SRIVASTAVA (1979) }\end{array}$ \\
\hline $\begin{array}{l}\text { S. ferax } \\
\text { (S. mixta et } \\
\text { S. monoica) }\end{array}$ & $\begin{array}{l}\text { WALLENTOWITZ (1885), CLINTON (1894), SCOTT et O'BIER } \\
\text { (1962). ARASAKI (1958). NOLARD-TINTIGNER (1970) }\end{array}$ \\
\hline $\begin{array}{l}\text { S. parasitica } \\
\text { (Sensu KANOUSE) }\end{array}$ & $\begin{array}{l}\text { HOSHINA et al. (1960), SCOTT et O'BIER (1962), NEISH } \\
\text { (1977). SHAH et al. (1977) }\end{array}$ \\
\hline S. hypogyna & BOOTSMA (1973), BUCHE MAFF et al. (1979) \\
\hline
\end{tabular}

Tableau 2 : Espèces de Saprolegnia reconnues parasites lors de la saprolegniose expérimentale.

\begin{tabular}{|l|l|}
\hline $\begin{array}{l}\text { S. diclina } \\
\text { (S. delica) }\end{array}$ & $\begin{array}{l}\text { VISHNIAC et NIGRELLI (1957), SCOT et O'BIER (1968) } \\
\text { NOLARD-TINTIGNER (1970, 1973) }\end{array}$ \\
\hline \begin{tabular}{l} 
S. ferax $\begin{array}{l}\text { (S. mixta et } \\
\text { S. monoica) }\end{array}$ \\
\hline S. parasitica
\end{tabular} & $\begin{array}{l}\text { TIFFNEY (1939), VISHNIAC et NIGRELLI (1957), HOSHINA et } \\
\text { al. (1950), SCOT et O'BIER (1932). NOLARD-TINTIGNER } \\
(1970,1973)\end{array}$ \\
\hline SOSHINA et al. (1960), NOLARD-TINTIGNER (1973) \\
\hline S. megasperma
\end{tabular}

Tableau 3 : Inoculation de $S$. australis à deux espèces de poissons exotiques.

\begin{tabular}{|cc|c|c|c|}
\hline $\begin{array}{c}\text { Espèce et nombre } \\
\text { de poissons }\end{array}$ & $\begin{array}{c}\text { Mycose } \\
\text { evidente après }\end{array}$ & Mort après.. & $\begin{array}{c}\text { Aucune } \\
\text { infection }\end{array}$ \\
\hline $\begin{array}{c}\text { Lebistes } \\
\text { reticulatus }\end{array}$ & 2 & $12 \mathrm{~h}$ & $22 \mathrm{~h}$ & - \\
. & 2 & $12 \mathrm{~h}$ & $36 \mathrm{~h}$ & - \\
$\begin{array}{c}\text { Xiphophorus } \\
\text { helleri }\end{array}$ & 1 & $16 \mathrm{~h}$ & $72 \mathrm{~h}$ & 1 \\
\hline. & 1 & - & - & - \\
\hline
\end{tabular}

\section{- Transmission expérimentale}

Pour être certain que les souches isolèes représentent l'agent étiologique de la saprolegniose et non pas un coexistant saprophytique, nous avoins réalisé des essais d'inoculation expérimentale.

Les champignons utilisés proviennent toujours de cultures * bacieria free * et maintenues sur Corn-Meal-Agar Après le transfert dans les boites de Petri contenant de leau distillée stérile ainsi que des graines de chanvre, les souches sporulant abondamment sont utilisées comme matériel d'inoculation.

Les poissons expérimentaux sont des xiphos (Xiphosphorus helleri) et des guppys (Lebistes reticulatus). Ils sont préalablement baignés avec un fungicide, de préference le vert de Malachite. Ils sont ensuite anesthésiés par passage dans une solution de tricaine méthanosulfate (ou MS 222) à un pour mille ou avec l'ether monophénylique de l'éthylène-glycol 
à $0,3 \cdot 0,5 \mathrm{ml} / \mathrm{l}$. Les méthodes de l'inoculation par scarification ou voie intra-musculaire sont identiques à celles décrites par NOLARD-TINTIGNER (1970,1973). Les poissons traités sont remis en stabulation dans des aquariums dont la température est de $25^{\prime \prime} \mathrm{C} \pm 1$.

\section{RESULTATS}

\section{- Mycologie}

En culture sur graines de chanvre, les souches isolees forment des colonies de 2 à $3 \mathrm{~cm}$ de diamètre. Les filaments mycéliens sont denses avec des brindilles espacées. d'un diamètre de 15 à $40 \mu$ en moyenne et de $80 \mu$ à la base.

Les chlamydospores sont abondantes, de position tcrminale, assez souvent laterales. Elles sont rondes ou cylindriques, très souvent irrégulières. Ces organes se transforment parfois en oogones et plus fréquemment en sporanges.

Toutes les souches produisent des sporanges en abondance à température ambiante. Les sporanges croissent par proliferation interne. Ils sont filiformes ou cylindriques. Ils sont terminaux ou latéraux et mesurent en moyenne entre 350-650 $\mu$ de long (extrêmes 150 et $1200 \mu$ ) et $25-50 \mu$ de diamêtre (extrêmes 20 et $55 \mu$ ). La déhiscence des spores se fait par un orifice terminal et, pour les sporanges irrégulier's, par un ou plusieurs orifices latéraux. Quelquefois, la germination des spores se fait dans les sporanges comme chez le genre Aplanes.

Ce champignon a donné des oogones après une periode de quatre semaines, à température de $15: 1^{\circ} \mathrm{C}$. Elles sont terminales ou lateraies, quelquefois subterminales ou intercalaires. Plus ou moins sphériques, elles sont filiformes, fusiformes ou doliformes pour les intercalaires (Fig. 1). Quelquefois, elles se développent à la base d'un sporange vide ou d'une chlamydospore. Chez certaines souches, nous avons eu l'occasion de voir aussi des oogones avec des projections de quelques $u(i-10)$ qui contiennent des oospores (Fig. 2).

La paroi oogoniale, souvent irrégulière à sa surface interne, peut porter des proéminences parfois difficilement visibles. La tige qui supporte l'oogone, simple et solide, sou-

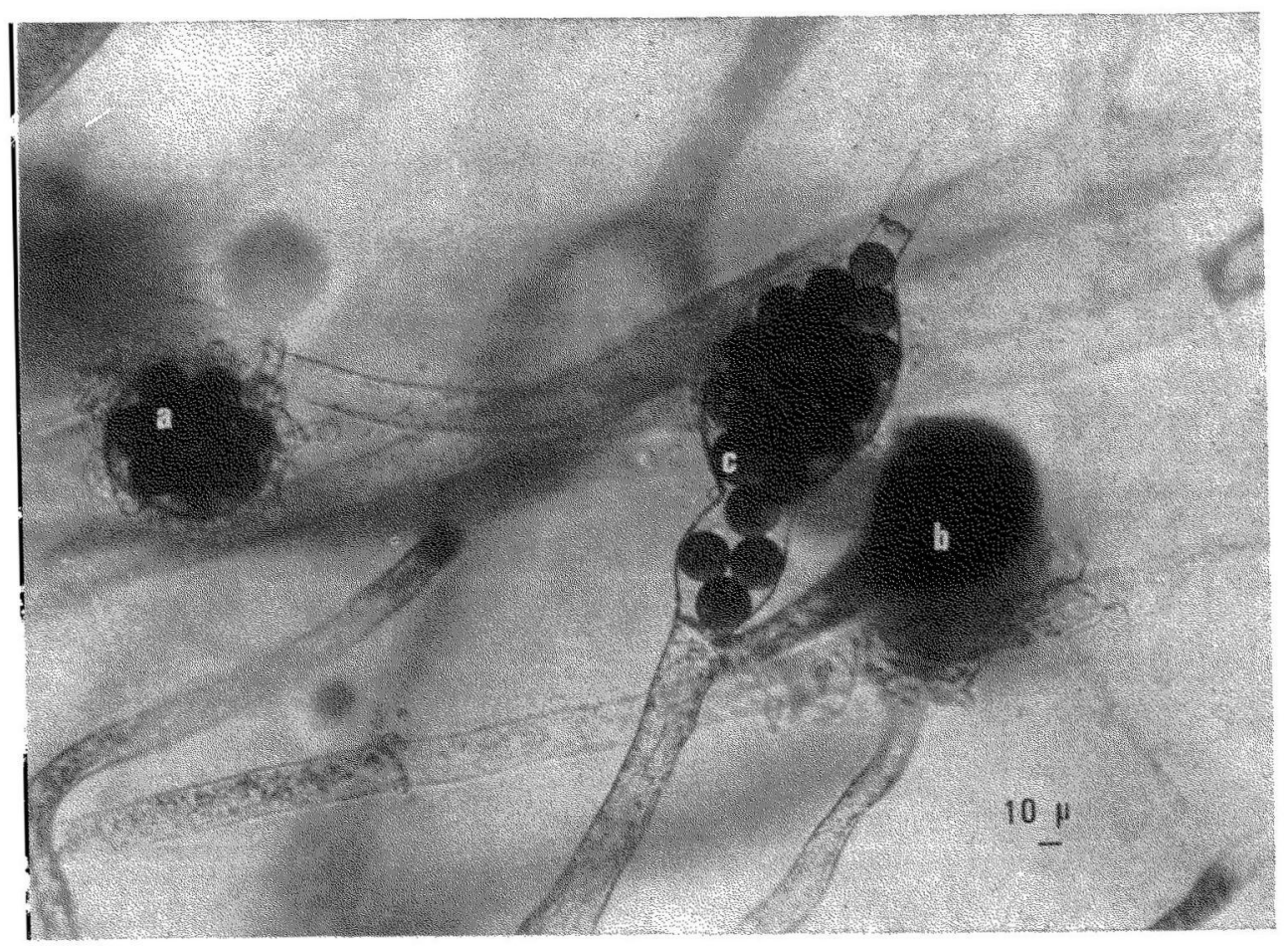

Fig. 1 : Oogones de Saprolegnia australis

a) Oogone terminale, vue de dessus.

b) Oogone latérale en formation (située dans un plan différent de celui de la photo).

c) Oogone intercalaire. 


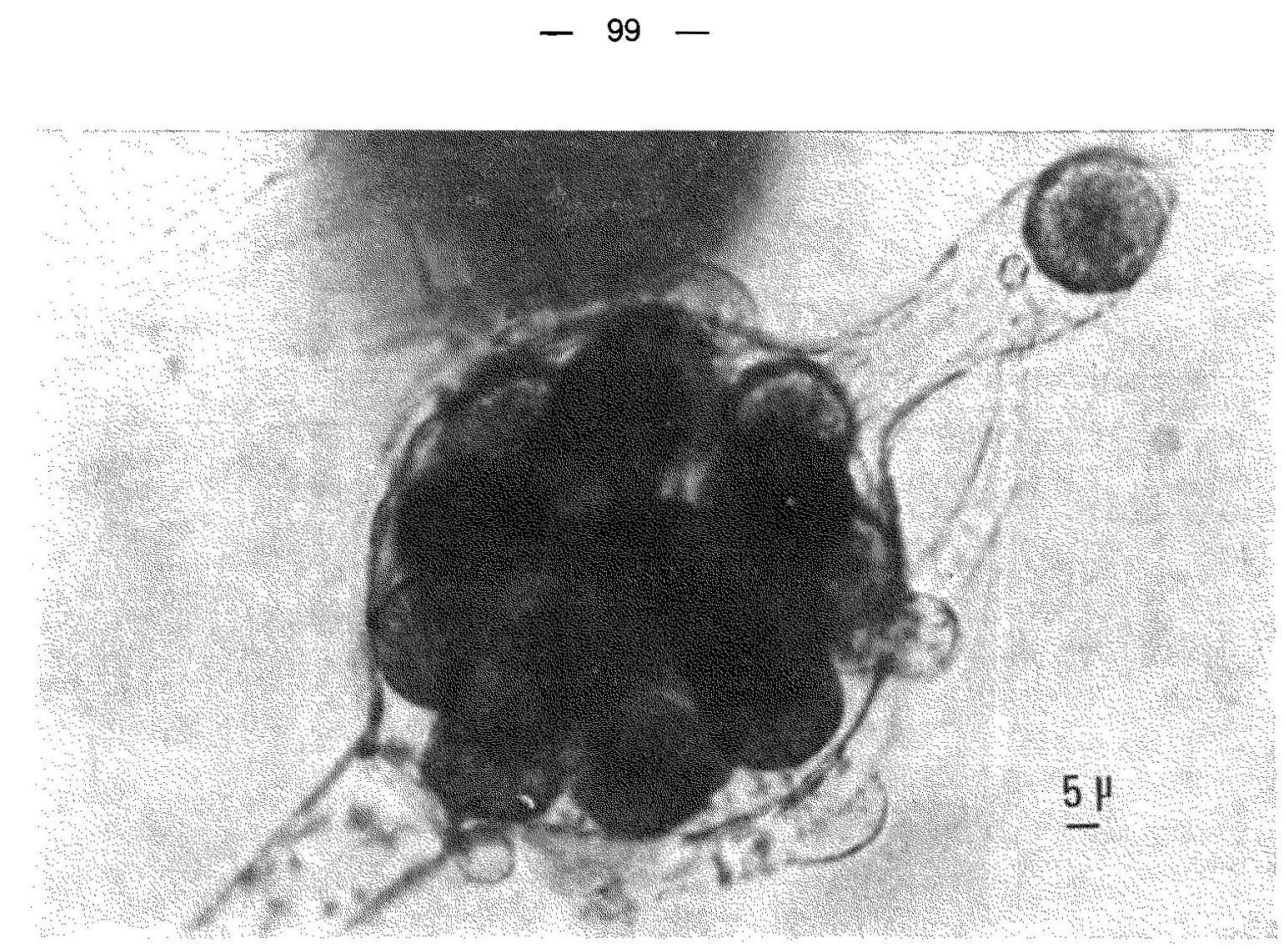

Fig. 2 : Oogone terminale avec projection contenant une spore. On remarque les enveloppements et projections de l'anthéridie.

vent longue, mesure habituellement au moins trois fois le cliamètre de l'oogone. Les oospores qui remplissent d'habitude l'oogone sont sphériques et occasionnellement elliptiques. Elles varient en nombre de 15 à 20 gènéralement (extrêmes $\hat{i}$ et 40 ) et en taille, 21 à $27 \mu$ de diamètre (extrêmes 10 et 30 ); la taille la plus fréquente est de 23 w. Elles sont centriques ou subcentriques. L'anthéridie dicline, rarement monocline, entoure souvent l'oogone et s'y applique lateralement ou avec des projections (Fig. 1-a et Fig. 2).

Cette étude des souches isolées nous a permis de determiner qu'il s'agissait de Saprolegnia australis Elliot, et de lui seul.

\section{- Transmission expérimentale}

Avec une seule souche bien sporulante, nous avons inoculé 4 guppys par scarification cutanée et 2 xyphos au moyen d'une poche intramusculaire. Nous avons observé, 8 à 12 heures après inoculation, l'apparition de filaments mycéliens sur les lésions artificielles (Fig. 3). La mort des poissons infectés survient en moins de 48 heures pour les guppys et en moins de trois jours pour les xyphos.

\section{DISCUSSION ET CONCLUSION}

Saprolegnia australis a èté récolté à l'ètat saprophyte en Nouvelle-Zèlande par ELLIOTT. Ce champignon n'a été signalè à l'ètat parasite qu'une seule fois. En effet, PICKERING et WILLOUGH8Y, en 1976, isolent une souche de S. austra'is en association avec d'autres champignons et principalement avec Leptomitus et Achlya qui se développaient sur les lésions de perches malades. Ce champignon a été airısi associé dans le * complexe fongique " des poissons, sans toutefois qu'on ait la preuve expérimentale de son pouvoir pathogène.

Après avoir isole $S$. australis en tant que parasite unique d'une saprolegniose spontanée, nous l'avons inoculé avec succès à des poissons exotiques. ce qui a permis de vérifier le pouvoir pathogene de cette espèce de champignon. L'utilisation de spores issues de souches sporulant en cultures stériles a permis la reussite de 5 inoculations sur 6 . ce qui suggère l'intérêt de determiner la dose infestante. Lorsque l'inoculation est réussie, elle est rapidement évidente et l'infestation est très vite généralisée. En effet, l'étude histologique des poissons expérimentaux montre la présence des hyphes mycéliens dans la musculature et les organes profonds, notamment autour et dans les vaisseaux principaux (aorte et veine) (Fig. 4). Il apparait ainsi que la mort rapide des sujets peut éventuellement survenir par thrombose occlusive. 


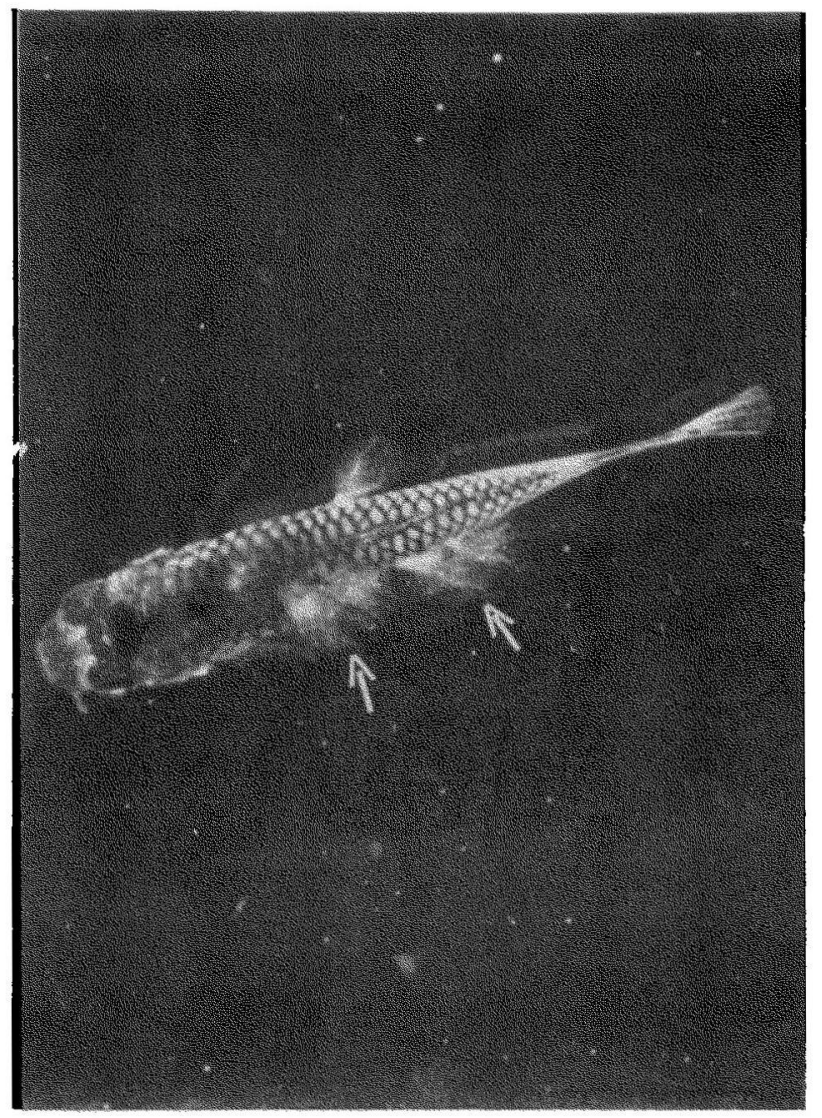

Fig. 3 : Etat d'un sujet (Lebistes) portant deux touffes de filaments mycéliens (flèches) 24 heures après inoculation expérimentale.



Fig. 4 : Coupe histologique dans le corps d'un Xiphophorus inoculé : hyphes mycéliens (flèches) autour des vaisseaux dorsaux et dans ceux-ci. 


\section{BIBLIOGRAPHIE}

BOOTSMA R., 1973. Infection with Saprolegnia in pike culture (Esox lucius L.) Aquaculture, 2. 385-394

BUCHE-MAFF D., CANLEY-MAFF G.D., GRAIG J.F., PICKERING A.P. et WILLOUGHBY L.G. 1979. Further studies of an epizootic of perch (Perca fluviatilis) of incertain aetiology. J. of Fish Diseases, 2, 297-311.

COKER W.C., 1923. The Saprolegniaceae. University of Nortin Carolina Press.

HOSHINA T., SANO T. et SYNAYAMA M., 1960. Studies on the saprolegniosis of eel. 1. Tokyo Univ. Fish., 47, 59-79.

HUXLEY T.H., 1882. Saprolegnia in relation to the Salmon disease. Quart. J. Microscop. Sci., 22, 311-333.

MURRAY G., 1885. Notes on the inoculation of fishes with Saprolegnia ferax. J. Bot., 23 , 302-308.

NOLARD-TINTIGNER N., 1970. Deux épidémies de saprolegriose des poissons par Saprolegnia ferax (Gruith) et par Saprolegnia diclina (Humphrey). Ann. Pars. Hum. Comp., 45, $761-770$.

NOLARD-TINTIGNER N., 1973. Etude expérimentale sur l'ecidémiologie et la pathogénie de la saprolegniose chez Lebistes reticulatus (Peters) fi Xiphophorus helleri (Hechel). Acta. Zool. Path. Antwerp., 57, 1-127.

PICKERING A.D. et WILLOUGHBY L.G., 1977. Epidermal lesions and fungal infection on the perch (Perca fluviatilis L.) in Windermere. J. Fish. Biol., 11, 349-359.

SCOTT W.N. et O'BIER A.H., 1962. Aquatic fungi associated with disease fish and fish eggs. Prog. Fish. Cultur., 24 (1), 3-15.

SCOTT W.N. et WARREN C.O. Jr., 1964. Studies of the host range and chemical control of fungi associated with diseased tropical fish. Virg. Agr. Exp. St. Techn. Bull., 171, Blackburg.

SEYMOUR R.L., 1970. The genus Saprolegnia. Nova Herwigia, XIX, 1-124. J. Cramer. Lehre.

SRIVASTAVA R.C. et SRIVASTAVA G.C., 1978 : Fungi associated with the diseases of freshwater fishes. Mycopathologia, 63 (2), 121-126.

TIFFNEY N.N., 1939. The identity of certain species of Saprolegniaceae parasitic to fish. J. Elisha Mitchell Sci. Soc., 55, 135-151.

VISHNIAC H.S. et NIGRELLI R.F., 1957. The ability of the Saprolegniaceae to parasitise platy fish. Zoologica, N.Y., 42, 131-134. 